Alterstice
Revue internationale de la recherche interculturelle
International Journal of Intercultural Research
Revista International de la Investigacion Intercultural

\title{
Compétence des nouvelles directions d'école de langue française au Canada pour la gestion inclusive de la diversité ethnoculturelle, linguistique et religieuse
}

\author{
Andréanne Gélinas Proulx, Claire IsaBelle et Hélène Meunier
}

Volume 4, numéro 1, 2014

URI : https://id.erudit.org/iderudit/1077483ar

DOI : https://doi.org/10.7202/1077483ar

Aller au sommaire du numéro

Éditeur(s)

Alterstice

ISSN

1923-919X (numérique)

Découvrir la revue

Citer cet article

Gélinas Proulx, A., IsaBelle, C. \& Meunier, H. (2014). Compétence des nouvelles directions d'école de langue française au Canada pour la gestion inclusive de la diversité ethnoculturelle, linguistique et religieuse. Alterstice, 4(1), 73-87.

https://doi.org/10.7202/1077483ar

\section{Résumé de l'article}

Compte tenu du changement démographique de la population scolaire, les écoles de langue française au Canada ont le mandat de favoriser une gestion inclusive de la diversité ethnoculturelle, linguistique et religieuse. Or, selon notre revue de la littérature, force est de constater que la compétence interculturelle des directions d'école pour assurer cette gestion inclusive de la diversité ne semble pas développée par certaines d'entre elles. Notre recherche qualitative menée auprès de 101 acteurs francophones de l'éducation des dix provinces canadiennes vise d'abord à présenter un portrait de l'état de la compétence interculturelle des directions d'école qui ont 5 ans et moins d'expérience en gestion scolaire (DÉ5-) en contexte francophone au Canada et, ensuite, à savoir si la compétence interculturelle devrait être inscrite dans les référentiels de compétences des directions d'école. Les résultats indiquent que les DÉ5- ne possèdent pas les indicateurs de compétence interculturelle requis pour assurer une gestion inclusive de la diversité, ce qui implique qu'elles tireraient des bénéfices d'une aide sous forme, entre autres, de formation. En outre, nous constatons un mutisme de la majorité des participants par rapport à cette compétence, que ce soit pour indiquer si elle est développée ou non chez les DÉ5- ou pour l'inscrire aux référentiels. De fait, nous recommandons qu'un travail de sensibilisation soit fait afin que les acteurs de l'éducation perçoivent la nécessité de cette compétence.
C Andréanne Gélinas Proulx, Claire IsaBelle et Hélène Meunier, 2014
Ce document est protégé par la loi sur le droit d'auteur. L’utilisation des services d’Érudit (y compris la reproduction) est assujettie à sa politique d'utilisation que vous pouvez consulter en ligne.

https://apropos.erudit.org/fr/usagers/politique-dutilisation/ 


\title{
7
}

ARTICLE HORS THÈME

\section{Compétence des nouvelles directions d'école de langue française au Canada pour la gestion inclusive de la diversité ethnoculturelle, linguistique et religieuse}

\author{
Andréanne Gélinas Proulx ${ }^{1}$, Claire IsaBelle ${ }^{2}$ et Hélène Meunier ${ }^{3}$
}

\section{Résumé}

Compte tenu du changement démographique de la population scolaire, les écoles de langue française au Canada ont le mandat de favoriser une gestion inclusive de la diversité ethnoculturelle, linguistique et religieuse. Or, selon notre revue de la littérature, force est de constater que la compétence interculturelle des directions d'école pour assurer cette gestion inclusive de la diversité ne semble pas développée par certaines d'entre elles. Notre recherche qualitative menée auprès de 101 acteurs francophones de l'éducation des dix provinces canadiennes vise d'abord à présenter un portrait de l'état de la compétence interculturelle des directions d'école qui ont 5 ans et moins d'expérience en gestion scolaire (DÉ5-) en contexte francophone au Canada et, ensuite, à savoir si la compétence interculturelle devrait être inscrite dans les référentiels de compétences des directions d'école. Les résultats indiquent que les DÉ5- ne possèdent pas les indicateurs de compétence interculturelle requis pour assurer une gestion inclusive de la diversité, ce qui implique qu'elles tireraient des bénéfices d'une aide sous forme, entre autres, de formation. En outre, nous constatons un mutisme de la majorité des participants par rapport à cette compétence, que ce soit pour indiquer si elle est développée ou non chez les DÉ5- ou pour l'inscrire aux référentiels. De fait, nous recommandons qu'un travail de sensibilisation soit fait afin que les acteurs de l'éducation perçoivent la nécessité de cette compétence.

\author{
Rattachement des auteures \\ ${ }^{1}$ Université du Québec en Outaouais, campus de Gatineau, Gatineau (Québec), Canada \\ ${ }^{2}$ Université d’Ottawa, Ottawa (Ontario), Canada \\ ${ }^{3}$ Université du Québec à Montréal, Montréal (Québec), Canada
}

\section{Correspondance}

andreanne.gelinas-proulx@uqo.ca

\section{Mots clés}

direction d'école; diversité; gestion inclusive; compétence interculturelle

\section{Pour citer cet article}

Gélinas Proulx, A., IsaBelle, C. et Meunier, H. (2014). Compétence des nouvelles directions d'école de langue française au Canada pour la gestion inclusive de la diversité ethnoculturelle, linguistique et religieuse. Alterstice, 4(1), 73-88. 


\section{Contexte et problématique}

Selon un rapport de l'Organisation de coopération et de développement économiques (OCDE, 2012), près de $20 \%$ de la population canadienne en 2010 est née à l'extérieur du pays. Ces immigrants de première génération se sont établis dans les dix provinces et les trois territoires canadiens, mais certaines provinces attirent davantage d'immigrants. Le tableau 1 présente la répartition géographique des immigrants ${ }^{1}$ au Canada selon l'Enquête nationale auprès des ménages de 2011 (Statistique Canada, 2014).

Dans cet article, nous nous intéressons plus particulièrement aux communautés francophones du Canada qui accueillent des nouveaux arrivants. À titre indicatif, dans la province de Québec en 2011, $87 \%$ de la population parle français à la maison (Statistique Canada, 2011, p. 2). Dans les autres provinces et territoires, de 0,8 \% à 33,2 \% de la population parle français à la maison (Statistique Canada, 2011). Le tableau 1 permet de voir où sont établis les immigrants francophones au Canada. Ceux-ci ont déclaré le "français " ou "l'anglais et le français " comme étant les premières langues officielles canadiennes parlées, c'est pourquoi nous supposons qu'ils ont rejoint une communauté francophone au Canada. Cette réalité démographique modifie également le tissu social des écoles de langue française au Canada financées par les deniers publics (Association canadienne d'éducation de langue française [ACELF], 2008).

Tableau 1. Répartition géographique des immigrants au Canada selon l’Enquête nationale auprès des ménages de 2011

\begin{tabular}{|c|c|c|c|}
\hline Provinces et territoires & $\begin{array}{c}\text { Nombre } \\
\text { d'immigrants }^{1}\end{array}$ & $\begin{array}{c}\text { Nombre } \\
\text { d'immigrants } \\
\text { francophones }\end{array}$ & $\begin{array}{l}\text { Pourcentage d'immigrants } \\
\text { francophones par rapport à } \\
\text { l'ensemble des immigrants (\%) }\end{array}$ \\
\hline Québec & 974895 & 664805 & 68,1 \\
\hline Ontario & 3611365 & 104760 & 2,9 \\
\hline Colombie-Britannique & 1191875 & 21230 & 1,8 \\
\hline Alberta & 644115 & 16235 & 2,5 \\
\hline Manitoba & 184505 & 3960 & 2,1 \\
\hline Nouveau-Brunswick & 28465 & 3720 & 1,3 \\
\hline Nouvelle-Écosse & 48270 & 1780 & 3,7 \\
\hline Saskatchewan & 68775 & 1475 & 2,1 \\
\hline Terre-Neuve-et-Labrador & 9160 & 210 & 2,3 \\
\hline Yukon & 3755 & 180 & 4,8 \\
\hline Île-du-Prince-Édouard & 7090 & 165 & 2,3 \\
\hline Territoires du Nord-Ouest & 2880 & 130 & 4,5 \\
\hline Nunavut & 610 & 25 & 4,1 \\
\hline
\end{tabular}

Source : Statistique Canada (2014)

Au Québec, depuis l'adoption de la Loi 101 en 1977, les enfants issus de l'immigration ont l'obligation de fréquenter l'école de langue française, sauf quelques exceptions (voir les articles 73, 76, 81, 85, 86 de la Charte de la langue française, Gouvernement du Québec, 2014), alors qu'auparavant ils pouvaient être inscrits à l'école de langue anglaise (Mc Andrew et Audet, 2010).

Hors Québec, les jeunes issus de l'immigration peuvent être inscrits à l'école de langue anglaise (avec ou sans programme d'immersion française) ou de langue française. Cependant, l'admission à l'école de langue française en

\footnotetext{
${ }^{1}$ « Immigrant désigne une personne qui est ou qui a déjà été un immigrant reçu/résident permanent. II s'agit d’une personne à qui les autorités de l'immigration ont accordé le droit de résider au Canada en permanence. Certains immigrants résident au Canada depuis un certain nombre d'années, alors que d'autres sont arrivés récemment. Certains immigrants sont citoyens canadiens, d'autres non. La plupart des immigrants sont nés à l'extérieur du Canada, mais un petit nombre d'entre eux sont nés au Canada. Dans l'Enquête nationale auprès des ménages de 2011, "immigrants" comprend les immigrants arrivés au Canada avant le 10 mai 2011. » (Statistique Canada, 2014).
} 
contexte minoritaire (milieu de valorisation culturelle et linguistique) n'est pas automatique, car plusieurs d'entre eux ne sont pas considérés comme des " ayants droit » à l'éducation en français selon l'article 23 de la Charte canadienne des droits et libertés (Émond, n. d.). De fait, ils peuvent avoir à passer par un comité d'admission pour démontrer leur intérêt vis-à-vis de la langue française et leur capacité à la comprendre et à s'exprimer, et ce, pour être inscrits (Émond, n. d.).

Tout compte fait, un nombre considérable d'élèves issus de l'immigration ont été accueillis dans les écoles de langue française, ce qui fait accroître sa diversité ethnoculturelle, linguistique et religieuse. Par exemple, en 20062007, 143 pays étaient représentés par les élèves fréquentant les écoles de langue française en Ontario (Ministère de l'Éducation de l'Ontario [MÉO], 2009a, p. 7). Cette diversité existe également chez le personnel de l'école et parmi ceux qui souhaiteraient y travailler (Pour parler profession, 2007).

Vis-à-vis de ce changement démographique de la population scolaire, les écoles de langue française devraient favoriser une gestion inclusive de la diversité (IsaBelle, Weatherall et Gélinas Proulx, 2011; Moisset, 2011), c'est-àdire mettre en place des pratiques d'intégration et non d'assimilation (Gérin-Lajoie et Jacquet, 2008), répondre aux besoins de tous les élèves, développer leur potentiel et éliminer la discrimination (Organisation des Nations Unies pour l'éducation, la science et la culture [UNESCO], 2008 et 2009). Ultimement, cela pourrait favoriser la réussite de tous les élèves. Dans cet ordre d'idées, les ministères de l'éducation du Canada ont développé des politiques et des programmes pour promouvoir cette inclusion de la diversité dans les écoles. Cependant, les interventions ne sont pas identiques puisque, l'article 93 de l'Acte de l'Amérique du Nord britannique (1867) stipule que l'éducation est une responsabilité provinciale. Ainsi, chaque province et territoire gère son système d'éducation, ce qui laisse place à une variété de politiques en matière d'inclusion de la diversité dans les écoles. À titre d'exemple, le ministère de l'Éducation de l'Ontario (MÉO, 2009c) a lancé une stratégie d'équité et d'éducation inclusive à laquelle s'arrime, entre autres, la politique d'admission, d'accueil et d'accompagnement dans les écoles de langue française de I’Ontario (MÉO, 2009a). Au Québec, la politique d'intégration scolaire et d'éducation interculturelle est en vigueur depuis 1998 (MÉQ) et en Colombie-Britannique, il existe un document-cadre du ministère de l'Éducation (2008) portant sur la diversité dans les écoles.

Or, selon notre recension des écrits en contexte anglodominant canadien et américain, au Québec et en contexte francophone de valorisation culturelle et linguistique au Canada (Archambault et Garon, 2013; Berger et Heller, 2001; Bouchamma et Tardif, 2011; Bustamante, Nelson et Onwuegbuzie, 2009; Cooper Wilson, 2009; El Ganzoury, 2012; Ryan, 2003), même si ces programmes existent, force est de constater que la compétence interculturelle (Cl) ou certains des indicateurs qui la composent ne semble pas développés par certaines directions d'école ${ }^{2}$, alors qu'elles devraient les posséder pour assurer une gestion inclusive de la diversité ethnoculturelle, linguistique et religieuse.

Il devient donc impératif d'avoir un portrait plus précis de l'état de la $\mathrm{Cl}$ des nouvelles directions d'école qui ont 5 ans et moins d'expérience en gestion scolaire (DÉ5-) en contexte francophone au Canada, d'une part, parce que le contexte démographique actuel exige une compétence de ce type et, d'autre part, parce que nous assistons à un renouvellement important des directions d'école depuis une décennie (Fortin, 2006; Gouvernement du Canada, 2013; Levine, 2005). II s'avère donc essentiel de pouvoir appuyer rapidement les DÉ5-. De fait, nous voulons savoir si les DÉ5- ont développé les indicateurs de la $\mathrm{Cl}$ pour gérer inclusivement la diversité ou si elles ont besoin d’aide dans les tâches qui demandent de déployer une telle compétence, c'est-à-dire si elles ont besoin de consolider leur $\mathrm{Cl}$. Cela permettrait d'ajuster l'offre de formation en administration scolaire pour favoriser l'inclusion de la diversité et la réussite scolaire des élèves.

De surcroît, pour encourager les directions d'école à démontrer une $\mathrm{Cl}$ et pour justifier l'offre de formation pour développer cette compétence, il serait souhaitable que les conseils scolaires ${ }^{3}$ et les écoles la priorisent et que les référentiels de compétences des directions d'école l'incorporent. Mais est-ce le cas, et est-ce souhaité par les

\footnotetext{
${ }^{2}$ Dans cet article, le terme « direction d'école » fait autant référence aux directions qu'aux directions adjointes d'école.

${ }^{3}$ Dans cet article, le terme " conseil scolaire » fait référence autant aux commissions scolaires, aux districts scolaires et aux divisions scolaires qu'aux conseils scolaires.
} 
acteurs scolaires? Parmi les documents ministériels des provinces et territoires, actuellement, nous n'avons repéré qu'un référentiel faisant explicitement état de la $\mathrm{Cl}$. Il s'agit du document Un personnel qui se distingue! Profil d'enseignement et de leadership pour le personnel des écoles de langue française de l'Ontario : guide d'utilisation qui stipule que le personnel des écoles de langue française devrait développer le cinquième champ de compétence intitulé Diversité des langues et cultures, c'est-à-dire qu'il devrait: "connaître, utiliser et apprécier les deux langues officielles. Reconnaître, mettre en valeur et apprécier la diversité des langues et cultures dans une optique d'ouverture interculturelle " (MÉO, 2011, p. 30). Par ailleurs, quelques référentiels traitent de cette compétence indirectement : ils incorporent certains indicateurs de la $\mathrm{Cl}$ à même d'autres compétences (British Columbia Principals' and Vice-Principals' Association [BCPVPA], 2013; Institut de leadership en éducation de l'Ontario, 2008; MELS, 2008; ministère de l'Éducation de l'Alberta, 2009). Par exemple, dans le Cadre de leadership pour les directions et directions adjointes des écoles de langue française : pratiques et compétences en leadership, il est mentionné que la direction doit "établir les orientations » de l'école et pour ce faire elle doit posséder l'attitude suivante: "est déterminée à instaurer une culture scolaire inclusive, respectueuse et équitable " (Institut de leadership en éducation de I'Ontario, 2008, p. 10). Enfin, d'autres référentiels n'abordent pas du tout cette compétence (Ministère de l'Éducation du Nouveau-Brunswick, 2005).

\section{Questions de recherche}

À la lumière de cette problématique, nos questions de recherche concernant la gestion inclusive de la diversité dans les écoles de langue française au Canada sont :

1. Dans quelle proportion différents acteurs de l'éducation croient-ils que les DÉ5- possèdent les indicateurs de la $\mathrm{Cl}$ et de quels indicateurs est-il question?

2. Dans quelle proportion différents acteurs de l'éducation mentionnent-ils que les DÉ5- ont besoin d'aide dans ces tâches qui demandent une $\mathrm{Cl}$ et quels indicateurs de la $\mathrm{Cl}$ ont été identifiés comme n'étant pas développés?

3. Dans quelle proportion les DÉ5- croient-elles que la $\mathrm{Cl}$ (ou certains de ces indicateurs) est priorisée par leur conseil scolaire et dans quelle proportion priorisent-elles cette compétence pour leur école?

4. Dans quelle proportion des acteurs de l'éducation autres que les DÉ5- croient-ils que la $\mathrm{Cl}$ (ou certains de ces indicateurs) devrait se retrouver dans un référentiel de compétences des directions d'école?

Nous utilisons pour y répondre le modèle hypothétique de la Cl développé par Gélinas Proulx (2014).

\section{Cadre conceptuel}

Le modèle hypothétique des composantes et des indicateurs visés de la $\mathrm{Cl}$ des directions et futures directions d'école de langue française au Canada en milieu de valorisation culturelle et linguistique pour favoriser la réussite de tous les élèves, de Gélinas Proulx (2014), a été développé à partir d'une étude menée auprès de six directions et d'une future direction d'école. Gélinas Proulx définit la $\mathrm{Cl}$ comme étant la capacité :

\footnotetext{
de se définir et d'identifier ses appartenances (fonction ontologique de la culture [qui comprend les éléments suivants : la famille, le langage, la communication, la religion, le gouvernement, la politique, l'éducation, la technologie, la société, le climat, la topographie et les systèmes économiques]). Elle permet également d'établir des relations avec des personnes porteuses de cultures dynamiques qui nécessitent de part et d'autre un ajustement constant et une communication adaptée (fonction instrumentale de la culture). Une adaptation, une transformation et une intercompréhension résultent dès lors de ces relations qui doivent aussi être caractérisées par l'inclusion, l'équité, la justice et la cohésion sociales. Ce savoir-agir nécessite donc que les directions d'école mobilisent de manière intériorisée des ressources lors de tâches interculturelles complexes en contexte scolaire. (p. 52)
}

La Cl comprend trois composantes, qui constituent donc les ressources à mobiliser, et qui se subdivisent en 12 indicateurs (figure 1). La composante "attitudes" comprend trois indicateurs: la conscience de soi (se connaître ou connaître, entre autres, ses biais, ses valeurs, son "niveau " de $\mathrm{Cl}$; le respect de soi et de ses cultures), l'ouverture (l'ouverture à la diversité ethnoculturelle, linguistique et religieuse; le respect des autres; la motivation à l'apprentissage interculturel; le changement de ses perceptions comme ses préjugés; l'attachement, 
l'amitié, le sentiment de proximité envers des gens d'autres cultures; la tolérance à l'ambiguïté) et le sentiment d'autoefficacité par rapport à la diversité. La composante " connaissances » comporte des savoirs : sur différentes cultures, sur différents systèmes d'éducation, pour soutenir les enseignants (connaissances sur des approches pédagogiques telles que les éducations interculturelle, multiculturelle, critique, antiraciste, à la citoyenneté, inclusive) et sur les théories du domaine de l'interculturel et de la justice sociale (comme les rapports de pouvoir, l'équité, l'ethnorelativisme, les mécanismes d'exclusion tels que les racismes, la culture et les modèles d'insertion des immigrants). La composante " habiletés » est constituée de cinq indicateurs : mettre en pratique des stratégies d'apprentissage d'une autre culture (observer, écouter, questionner et discuter), analyser des situations pour voir l'universalité et la diversité de même que s'autoanalyser, s'adapter (se décentrer, démontrer de l'empathie, modifier son agir et sa communication), inclure (répondre aux besoins des immigrants, ouvrir sa communauté à la diversité, combattre la discrimination) et réaliser l'une des missions de l'école de langue française, soit assurer la vitalité et la pérennité de la langue, et ce, tout en réussissant à faire une place pour tout le monde. En outre, il existe des interactions entre les indicateurs des composantes de la compétence, qui sont représentées par le diagramme de Venn. La flèche symbolise le développement progressif de la $\mathrm{Cl}$ à la suite d'expériences interculturelles variées et de plus en plus complexes. Enfin, il appert que les indicateurs se développent à des rythmes variables, c'est pourquoi les bulles du modèle peuvent être de tailles variables.

\section{Figure 1. Modèle hypothétique des composantes et des indicateurs visés de la compétence interculturelle des directions et futures directions d'école de langue française au Canada en milieu de valorisation culturelle et linguistique}

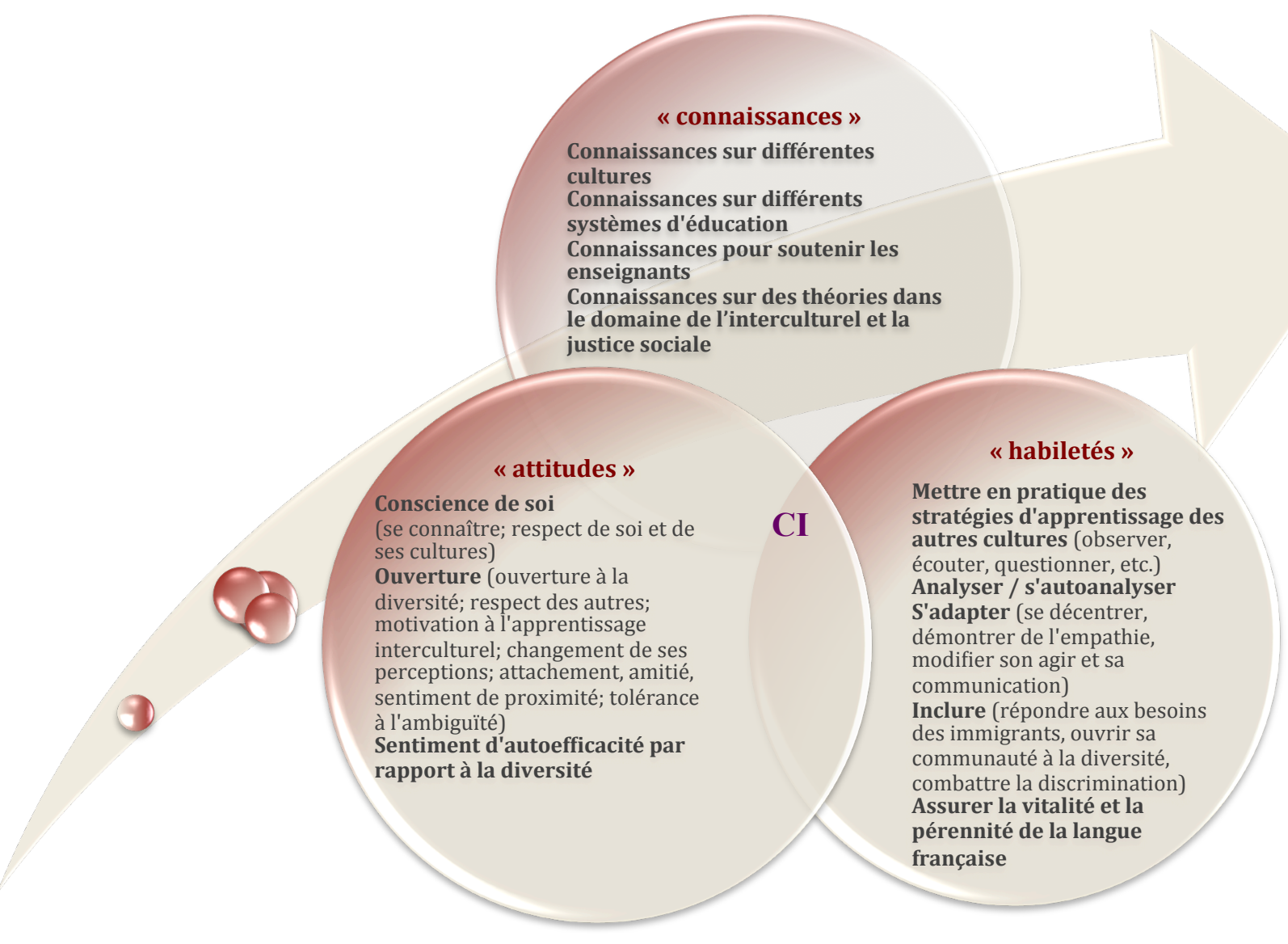

Source : adapté de Gélinas Proulx (2014).

Abréviation : $\mathrm{Cl}$, compétence interculturelle. 


\section{Méthodologie}

Notre étude sur la $\mathrm{Cl}$ des DÉ5- s'inscrit dans un programme de recherche plus large portant sur les contextes de formation et sur les compétences des nouvelles directions d'école de IsaBelle, Lapointe, Bouchamma, Clarke et Langlois (2005-2008). La première phase de ce programme de recherche reposait sur une démarche quantitative et visait spécifiquement les directions d'école travaillant en milieu francophone de valorisation culturelle et linguistique. Les directions du Québec n’ont donc pas participé à cette première phase, qui a été menée en 2006 auprès de 213 directions d'école de langue française à partir d'un questionnaire (IsaBelle et collab., 2008). La deuxième phase, dont la collecte de données s'est échelonnée de 2008 à 2009, s'inscrit dans un cadre qualitatif et interprétatif, car elle permet de mieux comprendre le sens des réponses données par les participants à partir du point de vue du chercheur (Savoie-Zajc, 2011). Cette deuxième phase, dont nous tirons les données présentées dans cet article, peut également déboucher sur des applications pratiques comme l'amélioration de l'offre de formation en administration scolaire (Savoie-Zajc, 2011). Nous la décrivons ci-dessous.

\section{Participants}

L'échantillon intentionnel (Savoie-Zajc, 2011) final comprend 101 acteurs de l'éducation francophone des dix provinces canadiennes : 30 directions d'école ayant 5 ans et moins d'expérience (DÉ5-), 28 directions d'école ayant 6 ans et plus d'expérience (DÉ6+), 20 directions générales ou adjointes de l'éducation de conseil scolaire (DGAÉCS), cinq présidents d'associations de directions d'école (PA) et 18 professeurs d'université (tableau 2). Leur participation s'est faite sur une base volontaire et ils ont tous signé un formulaire de consentement. Ayant une plus grande densité d'acteurs de l'éducation francophone au Québec, nous constatons qu'ils ont été également plus nombreux à participer.

\section{Tableau 2. Répartition des participants francophones selon leur lieu de travail et leur fonction professionnelle}

\begin{tabular}{|c|c|c|c|c|c|c|}
\hline Provinces & DGAÉCS & PA & PU & DÉ6+ & DÉ5- & Total \\
\hline Colombie-Britannique & 1 & - & 1 & 1 & 2 & 5 \\
\hline Alberta & 3 & - & - & 3 & 3 & 9 \\
\hline Saskatchewan & 1 & - & 1 & 1 & 3 & 7 \\
\hline Manitoba & 1 & & 1 & 2 & 2 & 6 \\
\hline Ontario & 4 & 1 & 1 & 9 & 5 & 20 \\
\hline Québec & 5 & 4 & 13 & 4 & 7 & 33 \\
\hline Nouveau-Brunswick & 2 & - & 1 & 2 & 3 & 8 \\
\hline Île-du-Prince-Édouard & 1 & - & - & 2 & 1 & 4 \\
\hline Nouvelle-Écosse & 1 & - & - & 2 & 3 & 6 \\
\hline Terre-Neuve-et-Labrador & 1 & - & - & 2 & 1 & 4 \\
\hline Total & 20 & 5 & 18 & 28 & 30 & 101 \\
\hline
\end{tabular}

Abréviations: DÉ, direction d'école; DGAÉCS, direction générale ou adjointe de l'éducation de conseil scolaire; PA, président d'association de directions d'école, PU, professeur d'université.

\section{Collecte de données}

Pour réaliser cette étude, les chercheurs IsaBelle, Lapointe, Bouchamma, Clarke et Langlois ont développé deux grilles d'entrevue. La première a été conçue pour les DÉ5- alors que la seconde s'adresse à tous les autres participants. Les grilles comprennent six sections : 1) identification du répondant; 2 ) les forces et les compétences que possèdent les DÉ5-; 3) les types de formation et les organismes ou groupes de personnes qui ont le plus aidé les DÉ5- à maîtriser leurs compétences; 4) les compétences que les DÉ5- doivent consolider et les tâches pour lesquelles elles ont besoin d'aide; 5) les types de formation et les organismes ou les groupes de personnes qui peuvent aider le plus les DÉ5- à développer les compétences qu'elles ne maîtrisent pas et 6) l'évaluation des compétences des directions d'école. Pour ajuster les grilles d'entrevue, une étude pilote a été réalisée auprès de quatre futures directions d'école. Les entrevues semi-dirigées (Savoie-Zajc, 2011) avec les participants de l'étude se sont déroulées dans leur milieu de travail respectif. Elles ont été réalisées par la chercheuse principale et des assistants de recherche. Elles ont été enregistrées et retranscrites. 


\section{Analyse des données}

La méthode d'analyse qualitative et inductive modérée a été employée pour analyser les données recueillies (Savoie-Zajc, 2011). Le logiciel NVivo 10 a été utilisé dans le but de faciliter la gestion des données et pour accomplir l'analyse. L'analyse s'est déroulée en huit temps : 1) un premier codage en parallèle a été fait avec les transcriptions des entrevues de 5 participants ayant des fonctions professionnelles différentes, et ce, par la chercheuse principale et deux assistantes de recherche; 2) pour comparer les codes émergents, il y a eu une discussion entre elles; 3) à partir du consensus sur les codes, un codage portant sur les entreveues avec 10 autres participants a été fait par les trois afin d'obtenir un accord interjuge satisfaisant, et cette étape a aussi permis de clarifier certains codes et d'ajouter celles qui ont émergé; 4) après le codage des entrevues avec 50 participants par une assistante, deux rencontres entre la chercheuse et l'assistante ont eu lieu pour condenser les codes (renommer, élargir, fusionner et éliminer des codes et sous-codes); 5) une assistante a ensuite codé les autres entrevues; 6) après le codage des 101 transcriptions d'entrevue, les quelques codes émergents ont été classifiés par la chercheuse et l'assistante qui se sont rencontrées à nouveau; 7) une dernière vérification a été faite par la chercheuse et quelques codes ont été modifiés par elle; 8) tous les codes se rapportant à l'inclusion de la diversité ont été sélectionné et leur contenu analysé pour cet article. Outre l'analyse qualitative décrite plus haut, nous avons également effectué un traitement quantitatif sur les données qualitatives, ce que Creswell (2009) appelle la transformation des données. Bien que l'étude soit avant tout qualitative, nous présentons dans la section suivante quelques données descriptives quantitatives.

Nous présenterons les résultats de notre étude en fonction de nos questions de recherche.

\section{Résultats}

\section{Indicateurs de la Cl possédés par les DÉ5-}

Dans cette section, nous répondons à la première question de recherche. À partir de l'analyse des données, il appert que peu de participants de notre étude mentionnent que les DÉ5- possèdent des indicateurs de la $\mathrm{Cl}$ pour assurer une gestion inclusive de la diversité. Seulement trois catégories d'acteur de l'éducation en font mention : les professeurs d'université, les DÉ5- et les DÉ6+. Ainsi, cinq (28\%) des professeurs interrogés sur les forces et les compétences des DÉ5- estiment que ces dernières possèdent des indicateurs de la $\mathrm{Cl}$. Un professeur du Manitoba mentionne par exemple :

j'ai l'impression qu'elles sont plus sensibilisées sur les obligations légales. Et, [ce n'est] pas seulement de suivre la loi scolaire et les politiques de la commission scolaire, mais [de connaître] la charte, les commissions des droits de la personne et les initiatives au niveau de la lutte contre la discrimination, les [formes d']aide [vis-à-vis de l'] immigration. (4d2.1)

Ce nombre chute à 2 (7\%) pour les DÉ5- et pour les DÉ6+. Une DÉ5- de la Saskatchewan affirme :

On a de plus en plus de gens qui nous arrivent de différentes provinces, mais aussi de différentes parties du monde. J'ai [des] connaissances. Moi, je suis très à l'aise là-dedans. (3f1.2)

et une DÉ6+ de l'Ontario précise :

C'est une qualité et une connaissance, mais ils ont un bagage multiculturel qui est plus large que [...] dans le passé. (5e1.5)

Enfin, aucune DGAÉCS et aucun PA ne mentionnent que les DÉ5- ont développé des indicateurs de la Cl.

Par ailleurs, le lieu de travail des participants qui ont émis des constats positifs sur le développement d'indicateurs de la $\mathrm{Cl}$ des DÉ5- est révélateur. Ils proviennent des provinces où le taux d'immigration francophone est le plus élevé, sauf pour le cas de la Saskatchewan (Statistique Canada, 2014) : 3 du Québec, 2 de I'Ontario, 1 de ColombieBritannique, 1 de l'Alberta, 1 du Manitoba et 1 de la Saskatchewan. 
Nous pouvons également identifier les indicateurs et sous-indicateurs de la $\mathrm{Cl}$ qui ont été ciblés par les participants. Parmi les attitudes, nous retrouvons l'ouverture à la diversité ( 3 mentions $)^{4}$, le respect des autres ( 1 mention) et le sentiment d'autoefficacité (1 mention) et, parmi les connaissances, celles des différentes cultures (2 mentions). En ce qui a trait aux habiletés, il est question d'analyser (collecter des données sur la situation des élèves issus de l'immigration et les analyser) (1 mention), de démontrer de l'empathie (1 mention), de répondre aux besoins des immigrants (2 mentions) et de combattre la discrimination (1 mention). Il appert que 6 indicateurs de la $\mathrm{Cl}$ n'ont pas été nommés. Par ailleurs, les propos de certains participants restent imprécis (3 cas) et ne nous permettent pas d'identifier un ou des indicateurs de la $\mathrm{Cl}$. Par exemple, une professeure du Québec mentionne :

[Elles possèdent une] compétence axée sur la diversité. (6d1.8)

À notre avis, cela pourrait vouloir dire qu'elles ont développé tous les indicateurs de la $\mathrm{Cl}$, mais cela ne peut être confirmé, car ces participants n'ont pas défini ce qu'ils entendaient par « compétence axée sur la diversité ».

À noter, 6 participants parmi les 9 tissent un lien entre les indicateurs de la Cl développés chez les DÉ5- et leurs expériences interculturelles antérieures, comme un processus d'immigration international ou interprovincial vécu, plusieurs voyages entrepris et une diversité côtoyée lors de leur scolarité. Une professeure de la ColombieBritannique explique :

Il y a certaines directions d'école qui viennent d'une francophonie internationale [...]. En fait, dans celles que je connais, [...], aucune n'est britannico-colombienne de souche. Donc, toutes ont fait le processus d'immigration qui est en soi [...] un avantage parce qu'[elles] reçoi[vent] des élèves qui sont dans le processus d'immigration donc [elles] sont capables de comprendre ce que les enfants et les familles sont en train de traverser. (1d1.1)

En outre, une DÉ6+ de l'Alberta mentionne à propos des DÉ5- :

Je pense que la plupart [...] elles ont beaucoup voyagé, elles ont une [...] ouverture sur le monde. Quand les gens arrivent de I'Afrique, [...] il y a beaucoup moins [...] de racisme. (2e1.2)

Ces résultats viennent confirmer que la $\mathrm{Cl}$ se développe par l'entremise d'expériences interculturelles (Bennett et Bennett, 2004; King et Baxter Magolda, 2005).

Nonobstant ces témoignages positifs sur les indicateurs de la $\mathrm{Cl}$ que les DÉ5- ont développés, il reste que cette perception n'est partagée que par 9 participants $(9 \%)$ de la recherche. Doit-on en conclure qu'une majorité de DÉ5- n'a pas développé une telle compétence? Dans la prochaine section de l'article, nous verrons justement que certains acteurs de l'éducation croient qu'elles ne l'ont pas développée, mais il reste que cela est aussi mentionné par peu de participants.

\section{Indicateurs de la $\mathrm{Cl}$ à consolider par les DÉ5-}

Dans cette section, nous répondons à notre deuxième question de recherche. Quelques participants $(9$, soit $9 \%)$ considèrent que les DÉ5- ont besoin d'aide dans les tâches qui demandent de gérer inclusivement la diversité. Nous retrouvons les mêmes catégories d'acteurs des mêmes provinces qui se sont prononcés sur le sujet, soit 5 professeurs (28\%), 2 DÉ5- (7\%) et 2 DÉ6+ (7\%). De fait, aucun PA et DGAÉCS n'estiment que les DÉ5- ont besoin d'aide dans cet aspect de leurs tâches. Par ailleurs, 4 participants qui ont mentionné que les DÉ5- possédaient des indicateurs de la $\mathrm{Cl}$ prétendent aussi qu'elles devraient recevoir de l'aide pour consolider cette compétence. Par exemple, une DÉ5- de l'Alberta témoigne :

je n'étais pas préparée à entrer en contact avec des Somaliens, avec des Congolais. [...] $95 \%$ de la population dans cette école est immigrante et chaque culture a ses particularités, ses approches, ses façons de faire, ses façons de s'habiller, ses façons d'agir, en couple, avec l'administration [et sa] vision de l'éducation au Canada. Alors ça, c'est un défi et j'apprends tous les jours. (2f1.3)

\footnotetext{
${ }^{4} \mathrm{Ce}$ chiffre indique le nombre de participants ayant identifié cet indicateur.
} 
Une DÉ6+ du Manitoba renchérit :

On a une grande population de nouveaux arrivants qui ne parlent même pas le français. [...] Et, c'est un défi pour ceux qui reçoivent un grand nombre de ces élèves. Alors, comment intégrer ces jeunes dans leur groupe âge? " (4e1.1)

Un professeur du Québec développe :

je disais qu'ils ont probablement la chance d'avoir côtoyé la diversité pendant qu'ils étaient élèves et même étudiants, mais ils ne sont pas nécessairement outillés [pour savoir] comment gérer, par exemple, les milieux défavorisés [et les] milieux réellement pluriethniques. Il y a certaines écoles où la très grande majorité [...] des élèves provient d'horizons différents. On ne peut pas tout connaître ces horizons-là, mais il faut avoir des approches, à tout le moins développer l'intérêt pour mieux connaître justement cette diversité et, en même temps, prendre aussi les moyens pour favoriser l'intégration de ces enfants, de ces jeunes et également du personnel. (6d2.13)

En ce qui a trait aux indicateurs de la $\mathrm{Cl}$ ciblés par les propos des participants qui estiment que les DÉ5- ont besoin d'aide dans les tâches en lien avec la gestion inclusive de la diversité, nous retrouvons, parmi les attitudes, le respect de soi et de ses cultures (1 mention), l'ouverture à la diversité (1 mention) et la motivation à l'apprentissage interculturel (1 mention). Des connaissances ont aussi été identifiées: les connaissances sur différentes cultures, et cela inclut les connaissances sur les parcours migratoires ( 3 mentions), les connaissances pour soutenir les enseignants (1 mention) et les connaissances sur les théories du domaine de l'interculturel et de la justice sociale (1 mention). En ce qui concerne les habiletés, les participants ont mentionné que les difficultés sont de répondre aux besoins des immigrants, ce qui inclut également de résoudre des conflits de valeurs (4 mentions), d'ouvrir sa communauté à la diversité (2 mentions) et de modifier son agir et sa communication (1 mention). Certains commentaires (3) sont imprécis et ne nous permettent pas d'identifier des indicateurs comme ceux d'une professeure du Québec :

La problématique de la diversification culturelle parmi la population étudiante et les parents, ça aussi c'est un aspect qui devrait être consolidé. (6d1.9)

De fait, il est peut-être nécessaire d'aider les DÉ5- à développer tous les indicateurs de la Cl par l'entremise d'une formation.

\section{Prioriser la Cl au conseil scolaire et à l'école}

Dans cette section, nous répondons à notre troisième question de recherche. Nous nous appuyons sur les réponses des DÉ5- à deux questions d'entrevue. La première question consistait à nommer au moins trois compétences chez une nouvelle direction d'école parmi celles qui sont les plus recherchées (priorisées) par leur conseil scolaire. La deuxième question visait à nommer au moins trois compétences principales qu'elles priorisent pour bien gérer leur école. À la première question, 2 DÉ5- (7\%) ont fait référence à un indicateur de la $\mathrm{Cl}$. Ces dernières proviennent de l'Ontario, ce qui n'est pas étonnant puisqu'il s'agit de la province canadienne qui accueille le plus d'immigrants francophones après le Québec (Houle et Corbeil, 2010; Statistique Canada, 2014). Qui plus est, dans le Cadre de leadership, certains indicateurs de la $\mathrm{Cl}$ (ouverture, connaissances pour soutenir les enseignants, inclure, s'adapter, assurer la pérennité et la vitalité de la langue française) y apparaissent (Institut de leadership en éducation de l'Ontario, 2008, p. 10 et 11) et, durant l'année de collecte des données, le ministère de l'Éducation de l'Ontario a produit plusieurs politiques en lien avec la gestion inclusive de la diversité (MÉO, 2009a, 2009b et 2009c). De fait, il semble que les contextes démographique et politique influencent les conseils scolaires à prioriser le développement de la $\mathrm{Cl}$ chez leur personnel. À titre d'exemple, une des DÉ5- de l'Ontario mentionne que son conseil scolaire accorde de l'importance aux directions d'école qui réalisent l'une des missions de l'école de langue française, soit d'assurer la vitalité et la pérennité de la langue. Dans l'optique de la $\mathrm{Cl}$, cet indicateur sous-entend que la direction fait la promotion du français tout en favorisant l'inclusion de la diversité. D'ailleurs, cette DÉ5soutient que son conseil scolaire priorise :

Un leadership communautaire aussi parce qu'il faut rassembler nos élèves [qui] viennent de partout. [...] le fait de promouvoir l'apprentissage en français, c'est ça qui est le plus important. (5f1.2) 
L’autre DÉ5- de l'Ontario précise que son conseil scolaire préconise une attitude d'ouverture à la diversité :

C'est certainement un personnel ouvert sur le monde. Notre conseil est tellement multiculturel que quelqu'un qui démontrerait des œillères, je ne pense pas qu'il serait embauché dans notre conseil. Non, c'est certain. (5f1.4)

Toutefois, nous insistons sur le fait que deux DÉ5- seulement se sont prononcées ce qui laisse sous-entendre que la $\mathrm{Cl}$ n'est pas priorisée par les conseils scolaires. Ce résultat est cohérent avec ceux obtenus aux deux premières questions de recherche, à savoir qu'aucune DGAÉCS n'a parlé de la Cl des DÉ5-.

Quant à la deuxième question d'entrevue, 2 DÉ5- (7\%) se sont prononcées et ont mentionné qu'elles priorisent un ou des indicateurs de la $\mathrm{Cl}$ dans leur école. Une DÉ5- de la Saskatchewan mentionne qu'elle priorise dans son école une attitude d'ouverture à la diversité, des connaissances pour soutenir les enseignants et une habileté à répondre aux besoins des élèves immigrants, ce qui inclut d'identifier et de comprendre leurs besoins :

Dans notre école, en particulier la question des cultures [... il faut] une sensibilité aux gens, de façon générale, aux enseignants, pour les accompagner dans un cheminement quand ils ont peur. Un cheminement professionnel et pour essayer de grandir dans les stratégies d'enseignement, dans les approches avec les jeunes [...]. C'est une sensibilité [aussi envers les] élèves. Tu as presque besoin d'avoir un sens, quand un jeune est préoccupé et tout ça, quand il agit de façon curieuse ou impolie, quelle est la raison? (3f1.2)

Une DÉ5- de l'Alberta mentionne pour sa part qu'elle priorise l'ouverture de sa communauté à la diversité :

Ça rentre dans mes valeurs au niveau académique, [de favoriser] l'ouverture d'esprit. On est une école [qui participe à un programme d'une organisation internationale] : ça ouvre l'esprit de nos enfants au niveau de ce qui se passe autour d'eux, les problèmes mondiaux, leur place dans la société. (2f2.2)

Nous constatons donc que les indicateurs de la $\mathrm{Cl}$ à prioriser dans leur école sont sensiblement les mêmes que ceux qui doivent être consolidés. De fait, ces indicateurs devraient éminemment être développés dans le cadre de formations.

\section{Inclure la $\mathrm{Cl}$ dans un référentiel de compétences pour les directions d'école}

Pour répondre à notre quatrième question de recherche, nous considérons les réponses de tous les acteurs de l'éducation exceptés les DÉ5- à la question d'entrevue portant sur les compétences prioritaires à inclure dans un référentiel pour les directions d'école. Ainsi seulement une professeure du Québec a-t-elle fait vaguement allusion à la $\mathrm{Cl}$ en parlant de la compétence éthique :

Il y a des enjeux, de la complexité, il y a de la diversité, tout ça peut être regroupé dans une compétence éthique qui peut aider les gestionnaires à faire de bons coups, à mieux analyser ses décisions, à mieux vérifier l'impact que cela peut avoir dans la communauté, dans la société. Donc, il y a une compétence éthique. (6d1.8)

En effet, nous supposons que pour analyser ses décisions en lien avec la diversité, il est bénéfique que la direction possède une $\mathrm{Cl}$. Bref, selon les résultats, il semble que la $\mathrm{Cl}$ ne constitue pas une compétence priorisée ou à prioriser, mais selon la littérature scientifique elle constitue une compétence à développer chez les directions d'école pour favoriser la réussite scolaire de tous les jeunes (Bennett, 2009; Lindsey, Robins et Terrell, 2009; Matthews et Crow, 2010; Mc Andrew, 2008; Ouellet, 2010). De plus, ce silence n'est pas justifié quand nous mettons en parallèle le contexte démographique des écoles de langue française au Canada. À notre sens, cette compétence, qui est transversale, aurait dû être mentionnée plus souvent puisque son déploiement préventif peut permettre d'éviter des problèmes tels que la discrimination, l'exclusion, les iniquités, les conflits interethniques et interreligieux et les échecs scolaires de certains groupes, problèmes qui demanderaient de façon réactive de démontrer d'autres types de compétence comme un leadership éducationnel, la gestion des ressources humaines, la gestion des conflits et la gestion du changement. De fait, la Cl devrait être davantage priorisée en amont. 


\section{Conclusion}

Nos résultats indiquent que l'ensemble des DÉ5- ne possède pas les indicateurs de la $\mathrm{Cl}$ requis pour assurer une gestion inclusive de la diversité ethnoculturelle, linguistique et religieuse dans les écoles de langue française au Canada, ce qui confirme les résultats d'autres études. Toutefois, selon le témoignage de quelques participants, les DÉ5- seraient mieux équipés en matière de gestion de la diversité que les directions en poste depuis plus longtemps. De fait, c'est peut-être l'ensemble des directions d'école qui devrait recevoir de l'aide pour développer les indicateurs des trois composantes de la $\mathrm{Cl}$. Une façon de les aider serait de leur proposer une formation qui tient compte de ces résultats et qui s'appuierait sur les éléments à incorporer dans une formation destinée à favoriser la justice sociale (Brown, 2004; McKenzie et collab., 2008). De plus, un certain type de formation pourrait être privilégié, à savoir un stage dans un autre pays, car il semble favoriser le développement des trois composantes de la Cl (Dinnan, 2009; Gélinas Proulx, 2014).

Même si les DÉ5- de l'ouest et du centre du Canada sont pour l'instant davantage concernées par le développement de la $\mathrm{Cl}$ parce que plus d'immigrants francophones sont accueillis dans ces régions (Statistique Canada, 2010 et 2014), il appert, selon Ouellet (2000), que :

[...] cette conception de l'éducation interculturelle [c'est-à-dire des initiatives éducatives qui visent selon lui l'équilibre entre l'ouverture à la diversité, l'égalité des chances et l'équité, tout comme la cohésion sociale] et de la formation dont ont besoin ceux et celles qui veulent la mettre en œuvre ne concerne pas seulement les écoles situées dans des secteurs où la diversité ethnique et religieuse est plus marquée, mais l'ensemble des écoles, y compris celles qui sont situées dans des communautés relativement homogènes sur les plans culturel, ethnique et religieux. (p. 377)

Dans cette optique, les DÉ5- de l'est du Canada devraient également se sentir concernées.

Quant au mutisme de la majorité des participants par rapport à cette compétence, que ce soit pour indiquer qu'elle est développée ou non chez les DÉ5- ou que ce soit pour l'inscrire aux référentiels, il nous laisse penser qu'un travail de sensibilisation doit être fait auprès de tous les acteurs de l'éducation afin qu'ils perçoivent que cette compétence est nécessaire pour gérer une école de langue française au Canada et pour favoriser la réussite de tous les élèves. Par conséquent, il serait pertinent d'inscrire explicitement la $\mathrm{Cl}$ aux référentiels de compétences des directions d'école, comme le recommande également Ouellet (2010). Selon nous, cela serait un premier pas vers une plus grande inclusion de la diversité si les référentiels pouvaient ensuite orienter les formations, l'accompagnement et la supervision des directions d'école. De plus, les DGAÉCS comme les PA sont probablement les premiers acteurs à devoir être sensibilisés, compte tenu de leur manque apparent d'intérêt pour la $\mathrm{Cl}$ et de leur position "d'autorité » vis-à-vis des directions d'école. Nous pouvons d'ailleurs supposer que les personnes qui occupent de tels postes, souvent d'anciennes directions d'école, n'ont jamais reçu de formation portant sur les préceptes de l'inclusion de la diversité. Cela ne serait pas étonnant, car une recension effectuée par Gélinas Proulx (2014) et Ouellet (2010) montrent que les cours sur ce sujet dans les programmes d'administration éducationnelle à l'université sont souvent optionnels, abordés rapidement au sein de cours plus généraux ou difficilement accessibles, voire non disponibles en français dans certaines provinces. De fait, comment auraient-elles pu parler de la $\mathrm{Cl}$ ou de ses indicateurs si elles ne sont pas au fait des implications de la diversité et si ce concept ne fait pas partie de leur vocabulaire? La place de ce thème dans la formation mériterait donc d’être révisée.

Notre recherche comporte certaines limites. D'abord, il aurait été souhaitable d'avoir une meilleure représentation de certains acteurs de l'éducation dans toutes les provinces, par exemple plus de professeurs en Ontario et quelques participants venant des trois territoires canadiens. Ensuite, ni les transcriptions d'entrevue ni les interprétations n’ont été vérifiées par les participants, ce que Gohier (2004) appelle la validité de signifiance des interprétations dans le but d'augmenter la rigueur scientifique de la recherche. Enfin, les données sont tirées d'une recherche plus large qui étudie l'ensemble des compétences des DÉ5- pour gérer l'école de langue française d'aujourd'hui, comme le leadership, la communication et la gestion des ressources humaines et matérielles. De fait, les participants n'ont pas eu, lors des entrevues, à donner de précisions sur les composantes et les indicateurs de la $\mathrm{Cl}$. Par conséquent, nous suggérons de mener une étude spécifique sur l'état de la $\mathrm{Cl}$ des DÉ5- ainsi que sur celle des futures directions d'école et des DÉ6+. En ce sens, notre recherche constitue une première étape dans le 
processus de comprenhension de l'état de la $\mathrm{Cl}$ des nouvelles directions d'école de langue française au Canada pour la gestion inclusive de la diversité ethnoculturelle, linguistique et religieuse.

\section{Remerciements}

Les auteures tiennent à remercier les participants de cette recherche, de même que le Conseil de recherches en sciences humaines (CRSH) pour leur subvention. Elles tiennent également à souligner le travail des cochercheurs, à savoir Claire Lapointe, Yamina Bouchamma, Lyse Langlois et Paul Clarke.

\section{Références bibliographiques}

Association canadienne d'éducation de langue française [ACELF] (2008). Carte des écoles francophones [en ligne]. http://www.acelf.ca/outils-pedagogiques/carte-ecoles-francophones.php

Archambault, J. et Garon, R. (2013). How principals exercise transformative leadership in urban schools in disadvantaged areas in Montréal, Canada. International studies in educational administration, 41(2), 49-66.

Bennett, J. (2009). Transformative training: Designing programs for culture learning. Dans M. A. Moodian (dir.), Contemporary leadership and intercultural competence: Understanding and utilizing cultural diversity to build successful organizations (p. 95-110). Thousand Oaks, CA : Sage.

Bennett, J. et Bennett, M. (2004). Developing intercultural sensitivity: An integrative approach to global and domestic diversity. Dans D. Landis, J. Bennett et M. Bennett (dir.), Handbook of intercultural training ( $3^{\mathrm{e}}$ éd., p. 147-165). Thousand Oaks, CA : Sage.

Berger, M.-J. et Heller, M. (2001). Promoting ethnocultural equity education in franco-ontarian schools. Language, Culture and Curriculum, 14(2), 130-141.

Bouchamma, Y. et Tardif, C. (2011). Les pratiques des directions d'écoles en contexte de diversité ethnoculturelle. Dans F. Kanouté et G. Lafortune (dir.), Familles québéquoises d'origine immigrante : les dynamiques de l'établissement (p. 87-96). Montréal : Presses de l'Université de Montréal.

British Columbia Principals' and Vice-Principals' Association [BCPVPA]. (2013). Leadership Standards for Principals and Vice-Principals in British Columbia [en ligne].

http://www.bcpvpa.bc.ca/downloads/pdf/Standardsfinal.pdf

Brown, K. (2004). Leadership for social justice and equity: Weaving a transformative framework and pedagogy. Educational administration quarterly, 40(1), 77-108.

Bustamante, R., Nelson, J. et Onwuegbuzie, A. (2009). Assessing schoolwide cultural competence: Implications for school leadership preparation. Educational administration quarterly, 45(5), 793-827.

Cooper Wilson, C. (2009). Performing cultural work in demographically changing schools: Implications for expanding transformative leadership frameworks. Educational administration quarterly, 45 (5), 694-724.

Creswell, J. (2009). Research design: Qualitative, quantitative, and mixed methods approaches ( $3^{\mathrm{e}}$ éd.). Thousand Oaks, CA : Sage.

Dinnan, P. (2009). The effects of a short-term cultural immersion experience to Mexico on school leaders (thèse de doctorat). Georgia State University, Atlanta, États-Unis. Pro Quest Dissertations and Theses, UMI $n^{\circ} 577393753$

El Ganzoury, H. (2012). Assessing Intercultural Competence for Educational Leaders: An Empirical Investigation. (thèse de doctorat). University of Minessota, Minneapolis, États-Unis. http://conservancy.umn.edu/bitstream/127965/1/EIGanzoury_umn_0130E_12671.pdf

Émond, G. (n. d.). Vivre ensemble dans les écoles de langue française : l'accueil des jeunes immigrantes et immigrants à l'école de la francophonie canadienne [en ligne]. http://www.ctffce.ca/publications/Frenquetes/Articl_Immigrants_Genevi\% C3\%A8ve_\%C3\%89mond.pdf 
Fortin, R. (2006). L'insertion professionnelle des nouvelles directions d'établissement d'enseignement. Rapport pour la Fédération québécoise des directeurs d'établissement. http://www.fqde.qc.ca/documents/AbregeVersPaysage.pdf

Gélinas Proulx, A. (2014). Modèles hypothétiques de la compétence et d'une formation interculturelles pour des directions et futures directions d'école de langue française au Canada (thèse de doctorat). Université d'Ottawa, Ontario, Canada. Base RUOR, http://www.ruor.uottawa.ca/fr/handle/10393/30655

Gérin-Lajoie, D. et Jacquet, M. (2008). Regards croisés sur l'inclusion des minorités en contexte scolaire francophone minoritaire au Canada. Éducation et francophonie, 36(1), 25-43.

Gohier, C. (2004). De la démarcation entre critères d'ordre scientifique et d'ordre éthique en recherche interprétative. Recherches qualitatives, 24, 3-17.

Gouvernement du Canada (2013). Explorer des carrières - rapport sur le marché du travail : directeurs/directrices d'école et administrateurs/administratrices de programmes d'enseignement aux niveaux primaire et secondaire (cnp 0313) [en ligne]. http://www.travailleraucanada.gc.ca/accueil-fra.do

Gouvernement du Québec (2014). Charte de la langue française (abrogé le $1^{\mathrm{er}}$ août 2014) [en ligne]. http://www2. publicationsduquebec.gouv.qc.ca/dynamicSearch/telecharge.php?type=2\&file=/C_11/C11.html

Houle, R. et Corbeil, J.-P. (2010). Portrait statistique de la population immigrante de langue française à l'extérieur du Québec (1991 à 2006) [en ligne]. Ottawa : Statistique Canada. http://www.statcan.gc.ca/pub/89-641x/89-641-x2010001-fra.pdf ( ${ }^{\circ} 89-641-X 2010001$ au catalogue)

Institut de leadership en éducation de l'Ontario. (2008). Mise en application du Cadre de leadership de l'Ontario. Guide pour les leaders scolaires et les leaders du système [en ligne]. http://live.iel.immix.ca/storage/2/1284580745/CadreLeadershipOntario.pdf

IsaBelle, C., Lapointe, C., Bouchamma, Y., Clarke, P., Langlois, L. et Leurebourg, R. (2008). Contextes de formation chez les directions d'écoles francophones au Canada. Revue de recherche appliquée sur l'apprentissage, 2(1), $1-20$.

IsaBelle, C., Weatherall, L. et Gélinas Proulx, A. (2011). Le dispositif VelTIC : Pour les leaders scolaires de la francophonie canadienne pluriethnique. Dans J. Rocque (dir.), La direction d'école et le leadership pédagogique en milieu francophone minoritaire (p. 159-176). Winnipeg : Presses universitaires de SaintBoniface.

King, P. et Baxter Magolda, M. (2005). A developmental model of intercultural maturity. Journal of college student development, 46, 571-592.

Levine, A. (2005). Educating School Leaders [en ligne]. http://www.edschools.org/pdf/Final313.pdf

Lindsey, R., Robins, K. et Terrell, R. (2009). Cultural proficiency: A manual for school leaders ( $3^{\mathrm{e}}$ éd.). Thousand Oaks, CA : Corwin Press.

Matthews, L. et Crow, G. (2010). The principalship: New roles in a professional learning community. Boston, MA : Prentice-Hall.

Mc Andrew, M. (2008). Une réflexion sur la formation des intervenants. Dans M. Mc Andrew, M. Milot, J.-S. Imbeault et P. Eid (dir.), L'accommodement raisonnable et la diversité religieuse à l'école publique : normes et pratiques : actes du Colloque « Prise en compte de la diversité à l'école publique » (p. 135-156). Québec, Canada : Fides.

Mc Andrew, M. et Audet, G. (2010). Trente ans d'adaptation institutionnelle à la diversité en milieu scolaire : bilan et défis. Nos diverses cités, 7, 129-135.

McKenzie, K., Christman, D., Hernandez, F., Fierro, E., Capper, C., Dantley, M., ... Scheurich, J. (2008). From the field: A proposal for educating leaders for social justice. Educational administration quarterly, 44(1), 111-138. 
Ministère de l'Éducation de la Colombie-Britannique. (2008). La diversité dans les écoles de la ColombieBritannique : document-cadre [en ligne]. http://www.bced.gov.bc.ca/diversity/f_diversity_framework.pdf

Ministère de l'Éducation de l'Alberta (2009). Guide pour une pratique de qualité à l'intention de la direction d'école : pour encourager le leadership scolaire en Alberta [en ligne]. http://education.alberta.ca/media/949133/principal-quality-practice-guideline-french-12feb09.pdf

Ministère de l'Éducation de l'Ontario [MÉO] (2009a). L'admission, l'accueil et l'accompagnement des élèves dans les écoles de langue française de l'Ontario. Énoncé de politique et directives [en ligne]. http://www.edu.gov.on.ca/fre/document/policy/admissions.pdf

Ministère de l'Éducation de l'Ontario [MÉO] (2009b). Une approche culturelle de l'enseignement pour l'appropriation de la culture dans les écoles de langue française en Ontario [en ligne]. http://www.edu.gov.on.ca/fre/amenagement/PourLaFrancophonie2009.pdf

Ministère de l'Éducation de l'Ontario [MÉO] (2009c). Comment tirer parti de la diversité. Stratégie ontarienne d'équité et d'éducation inclusive [en ligne]. http://www.edu.gov.on.ca/fre/policyfunding/equity.pdf

Ministère de l'Éducation de l'Ontario [MÉO] (2011). Un personnel qui se distingue! Profil d'enseignement et de leadership pour le personnel des écoles de langue française de l'Ontario - Guide d'utilisation [en ligne]. http://www.edu.gov.on.ca/fre/amenagement/teacher.html

Ministère de l'Éducation, du Loisir et du Sport [MELS] (2008). La formation à la gestion d'un établissement d'enseignement. Les orientations et les compétences professionnelles [en ligne]. www.mels.gouv.qc.ca/fileadmin/site_web/documents/reseau/formation_titularisation/07-00881.pdf

Ministère de l'Éducation du Nouveau-Brunswick (2005). Programme d'évaluation des directions d'école [en ligne]. http://www.gnb.ca/0000/publications/evalf/PED\%20-\%20version\%20finale\%20\%2029\%20septembre\%202005_WEB.pdf

Ministère de l'Éducation du Québec [MÉO] (1998). Une école d'avenir. Politique d'intégration scolaire et d'éducation interculturelle [en ligne]. Document téléchargeable à partir de la page http://www.mels.gouv.qc.ca/references/publications/resultats-de-la-recherche/detail/article/une-ecoledavenir-politique-dintegration-scolaire-et-deducation-interculturelle/

Moisset, J.-J. (2011). L'éducation interculturelle et la gestion scolaire : un modèle d'analyse. Dans J. Rocque (dir.), La direction d'école et le leadership pédagogique en milieu francophone minoritaire (p. 177-190). Winnipeg : Presses universitaires de Saint-Boniface.

Organisation de coopération et de développement économiques [OCDE] (2012). Effectifs de personnes nées à l'étranger dans les pays de l'OCDE et la Fédération de Russie [en ligne]. Données disponibles à partir de la page http://www.oecd.org/fr/els/politiquesetdonneesdesmigrationsinternationales/statcles.htm

Organisation des Nations Unies pour l'éducation, la science et la culture [UNESCO]. (2008). L'éducation pour l'inclusion : la voie de l'avenir. Rapport final de la $48^{e}$ session de la conférence internationale de l'éducation (ED/MD/104 CLD. 2447.9) [en ligne].

http://www.ibe.unesco.org/fileadmin/user_upload/Policy_Dialogue/48th_ICE/ICE_FINAL_REPORT_fre.pdf

Organisation des Nations Unies pour l'éducation, la science et la culture [UNESCO] (2009). L'éducation inclusive [en ligne]. http://www.unesco.org/fr/inclusive-education/

Ouellet, F. (2000). La formation interculturelle concerne-t-elle les écoles des milieux culturellement peu diversifiés? Cahiers de la recherche en éducation, 7(3), 375-406.

Ouellet, F. (2010). La prise en compte de la diversité en milieu scolaire. Dans P. Toussaint (dir.), La diversité ethnoculturelle en éducation : enjeux et défis pour l'école québécoise (p. 275-316). Québec: Presses de I’Université du Québec.

Pour parler profession (2007). Le marché du travail déçoit les enseignants néo-canadiens [en ligne]. http://pourparlerprofession.oeeo.ca/decembre_2007/transition_new-canadian.asp 
Ryan, J. (2003). Educational administrators' perceptions of racism in diverse school contexts. Race Ethnicity and Education, 6(2), 145-164.

Savoie-Zajc, L. (2011). La recherche qualitative/interprétative en éducation. Dans T. Karsenti et L. Savoie-Zajc (dir.), La recherche en éducation : étapes et approches ( $3^{\mathrm{e}}$ éd., p. 123-147). Saint-Laurent : ERPI.

Statistique Canada (2011). Le français et la francophonie au Canada : langue, recensement de la population de 2011 [en ligne]. http://www12.statcan.gc.ca/census-recensement/2011/as-sa/98-314-x/98-314-x2011003_1fra.pdf ( ${ }^{\circ}$ 98-314-X2011003)

Statistique Canada (2014). Certaines caractéristiques démographiques, culturelles, de la scolarité, de la population active et du revenu (730), première langue officielle parlée (4), groupes d'âge (8D) et sexe (3) pour la population du Canada, provinces, territoires, régions métropolitaines de recensement et agglomérations de recensement, Enquête nationale auprès des ménages de 2011 [en ligne]. Tableau consultable à partir de la page : http://www12.statcan.gc.ca/nhs-enm/2011/dp-pd/dt-td/Dir-fra.cfm (nº99-010-X2011043) 